\title{
1 Non-Uniform Transmission of Supermirror Devices for Neutron
}

\section{Polarization}

\author{
X. Tong ${ }^{\text {* }}$, J.L. Robertson ${ }^{1}$, R. Pynn ${ }^{1,2}$, \\ ${ }^{1}$ Instrument and Source Design Division, Oak Ridge National Laboratory, Oak Ridge, \\ TN, 37831, USA \\ ${ }^{2}$ Indiana University Center for the Exploration of Energy and Matter, Bloomington, IN, \\ 47408, USA
}

Abstract

Polarizing supermirrors have been widely used in neutron scattering facilities where they have been employed as neutron spin filters to polarize neutron beams as well as analyze their polarization. In the past, the performance of polarizing supermirrors has been limited by their small acceptance angle, which made them less suitable for use at short wavelengths or with highly divergent beams. Recent advances in supermirror coatings have led to an array of devices designed to, at least partially, overcome this limitation. V-polarizers and multichannel polarizers have been employed in several different types of neutron scattering instruments. However, our observations in the field where these types of polarizers are in use have raised concerns about their performance. In this paper, we report on detailed MonteCarlo simulations performed on a multi-channel polarizer used on a prototype Spin-Echo Small Angle Neutron Scattering (SESANS) instrument to better understand its performance. Our results show that careful simulations of polarizers based on mirror reflection are needed to determine whether a particular design is suitable for SESANS applications.

(C) 2014. This manuscript version is made available under the Elsevier user license http://www.elsevier.com/open-access/userlicense/1.0/ 


\section{I. Introduction}

The use of polarized neutrons provides an important capability for neutron scattering instruments because it allows, for example, separation of the nuclear and magnetic scattering cross-sections for both elastic and inelastic scattering ${ }^{1}$ or Larmor encoding of the neutron energy or momentum transfer ${ }^{2}$. In order to implement this capability, several different techniques have been developed to polarize neutron beams and to analyze their polarization.

Of these, the use of Heusler crystals ${ }^{3}$, polarized ${ }^{3} \mathrm{He}$ spin filters ${ }^{4,5}$, and polarizing supermirrors $^{6,7}$ are by far the most common. Heusler crystals diffract only one neutron spin state so they can be used to polarize the neutron beam or analyze the polarization of the neutron beam while simultaneously serving as a monochromator crystal or an analyzer

37 crystal to determine the energy transfer. Polarized ${ }^{3} \mathrm{He}$ spin filters, on the other hand, exploit the differential absorption cross sections for neutron spins that are parallel and antiparallel to the spin of the ${ }^{3} \mathrm{He}$ nuclei such that the neutrons with parallel spin are transmitted while those or analyze the neutron beam by reflecting only one of the neutron spin states and transmitting the other. Because supermirror polarizers offer high performance together with low maintenance, they are widely used ${ }^{12-14}$. 
In this paper we consider the performance of a multi-channel polarizer ${ }^{18}$ and its suitability for use on a Spin-Echo Small Angle Neutron Scattering (SESANS) instrument ${ }^{15,16}$. In particular, we have observed experimentally a spatial variation in the analyzing efficiency of such a device and in order to better understand the origins of this effect we have modeled the

behavior in detail. In the device we consider, neutrons are reflected from smooth mirror-like surfaces when their incident angle is less than the critical angle, $\theta_{c}$, for total external reflection. The critical angle depends on the neutron wavelength and the material the mirror is made from. Most materials have a refractive index that is slightly smaller than one for neutrons. For example the critical angle for a Ni mirror is given by $\theta_{c} \approx(0.1 \% \AA) \times \lambda$ where $\lambda$ is the neutron wavelength in angstroms. The effective critical angle can be increased beyond the total external reflection regime by employing a supermirror that consists of a multilayer coating on a smooth surface. The different layer materials are chosen to maximize the variation in the neutron scattering length density (SLD) giving rise to a continuum of overlapping Bragg scattering profiles which extend the reflectivity of the mirror out to the critical edge of the supermirrors. The critical edge of a supermirror depends on the range of multilayer layer thicknesses and the neutron wavelength while the reflectivity depends on the interface roughness and the difference in the SLD between the layers. In the case of a polarizing supermirror, the alternating layers of the multilayer coating typically comprise one material that is ferromagnetic and another that is nonmagnetic chosen such that the difference in SLD between the layers is large for one neutron spin state and small for the other spin state. This is possible because the SLD depends on both the nuclear, b, and magnetic, $p$, scattering lengths. In addition, the magnetic scattering length depends on the orientation of the magnetization vector in the material so that the total neutron scattering cross-section 
depends on $\varepsilon_{+}=b+p$ when the neutron spin is parallel to the magnetization and $\varepsilon_{-}=b-p$ when it is antiparallel. By a clever choice of multilayer materials the SLD in the magnetic layers for one spin state, usually $\varepsilon_{\text {, }}$, can match the SLD of the nonmagnetic layers resulting in a negligible Bragg scattering contrast for this spin state. As a consequence there is no extension of the mirrors reflectivity beyond the critical angle of the substrate $\left(\theta_{c}\right)$ for this spin state. On the other hand, the difference in SLD between the magnetic and nonmagnetic layers is $\sim 2 \mathrm{p}$, which can be quite large giving a high supermirror reflectivity extending from

$\theta_{\mathrm{c}}$ out to the supermirror critical edge. The net effect is that both spin states will be reflected below $\theta_{\mathrm{c}}$ but only one will be reflected between $\theta_{\mathrm{c}}$ and the critical edge of the supermirrors. Therefore, a polarizing supermirror can be used to polarize or analyze a neutron beam so long as all the incident angles are between $\theta_{c}$ and the supermirror critical edge. However, even though the critical edge for supermirrors has increased dramatically over the past 20 years ${ }^{17-19}$, this angle is still small even for cold (long wavelength) neutrons. Consequently, in order to polarize a relatively large neutron beam, an assembly of several supermirrors is required. Many supermirror geometries such as benders ${ }^{20,21}, \mathrm{~V}$-polarizers ${ }^{13}$, as well as multi-channel polarizers ${ }^{22}$ have been developed to achieve this goal. The polarizing-supermirror, multi-channel polarizer we simulate here consists of 17 channels, each only 3.13 millimeters wide. The central 9 channels are at $0^{\circ}$ to the axis of the device, while the upper and lower group of 4 channels is tilted in opposite directions by $0.1^{\circ}$.The parallel walls of each channel are Ni coated mirrors that have approximately the same critical angle for both neutron spin states. Diagonally across each channel is placed a polarizing supermirror made of alternating layers of Fe and Si deposited on a thin silicon wafer. Figure 1 shows a sketch of the two central channels of such a device. The Fe layers 
are magnetized so that the supermirrors reflect the up-spin state and transmit the down-spin

92 state. The nickel walls of the channel are designed to serve as a neutron guide and thus

93 increase the transmission of down-spin neutrons ${ }^{22}$ through the assembly. In order to inhibit

94 the transmission of neutrons between channels, the nickel layers on the channel walls are

95 backed by a neutron-absorbing material. Thus when the up-spin state is reflected from the

96 diagonal polarizing supermirror its trajectory will eventually intersect the channel wall with

97 an incident angle beyond the critical angle of nickel and it will be absorbed rather than

98 reflected.

99

100 Multi-channel polarizers of this type work well for neutron beams with relatively large cross-

101 sections (up to $30 \mathrm{~mm} \times 75 \mathrm{~mm}$ for the device considered here) and typically have polarizing

102 efficiencies greater than $90 \%$ over a broad range of neutron wavelengths. For many

103 experiments the polarizing efficiency is the primary reason for using this device. However,

104 for applications to Spin Echo Small Angle Neutron Scattering (SESANS) or, in some cases,

105 to measurements of diffuse magnetic scattering, the spatial and angular uniformity of the

106 polarizing efficiency and of the transmission efficiency are also quite important. In the

107 following sections we first consider quantitatively why the uniformity of both the

108 transmission and polarization analysis efficiencies is important in a SESANS experiment.

109 Next, we describe a series of computer simulations designed to model the performance of the

110 multi-channel polarizer we have used for our experiments in order to better understand the

111 variation in the down-spin transmission observed in the simulations.

112 We should stress that our detailed conclusions apply only to the device that we have

113 simulated. However, it becomes clear that any polarizing device that makes use of grazing 
incidence reflection of neutrons may potentially run into problems because multiple reflections can produce patterns of intensity that depend on the angle and position of a neutron striking the device. For reasons that we discuss in the next section, this implies that such devices need to be carefully simulated and/or measured before they are deployed for SESANS to ensure the absence of artifacts.

\section{Importance of Uniform Polarization Analysis for SESANS Measurements} SESANS uses Larmor precession ${ }^{15}$ to measure small changes of a neutron's direction of travel by coupling its trajectory to its spin polarization. SESANS is an attractive technique for some small angle scattering applications because it decouples instrumental angular resolution from the neutron wavelength spread and divergence of the neutron beam which translates into higher flux at the required resolution for certain classes of experiments ${ }^{15}$. Conceptually, a SESANS instrument consists of a polarizer; a spin-echo precession field whose borders are inclined relative to the beam by an angle $\theta_{0}$, the sample; a second spinecho precession field that is also inclined by $\theta_{0}$ but with the magnetic field in the opposite direction; a polarization analyzer ; and a neutron detector. See the instrument diagram in Fig.

2. Once the neutron beam is polarized, the polarization vector precesses around the field direction in the first spin-echo field, then if the neutron is not scattered by the sample the precession is reversed in the second spin-echo field and the neutron is returned to its original polarization state. This is independent of the neutron wavelength and direction of travel since the amount of precession is simply determined by the amount of time the neutron spends in each precession field. If, however, the propagation direction of the neutron changes when the neutron interacts with the sample, the path through the second spin-echo 
precession field will be different, i.e. the time within the precession field will be different, and therefore the neutron will not be returned to its original polarization state.

For a typical SESANS measurement, the instrumental polarization, $P_{0}$, of the neutron beam is first measured with either no sample or a non-scattering sample. Then the same measurement is repeated with the sample of interest in place and a new polarization, $P$, is measured for the beam after it has interacted with the sample. The key parameter to be two polarization measurements being made using an analyzer with uniform transmission and polarization efficiencies. As described in other work ${ }^{23}$, the ratio $\frac{P}{P_{0}}$ is a function of the spinecho length $z$ and gives a direct measurement of the normalized real-space correlation function $G(z)$ for the sample via the following equation:

$$
\frac{P(z)}{P_{0}}=\exp \left\{-s_{t}[1-G(z)]\right\}
$$

where $s_{t}$ is the total single-scattering cross section of the sample and $z$ is the spin-echo length for the architecture shown in Fig. 2 as given by:

$$
Z=\frac{c \lambda^{2} B L \cot \left(\theta_{0}\right)}{2 \pi}
$$

Where $c$ is the Larmor precession constant $\left(c=4.6368 * 10^{14} T^{-1} m^{-2}\right), \lambda$ is the neutron wavelength, $B$ is the magnetic field, $L$ is the length of the field coils and $\theta_{0}$ is the inclination angle of the field coils.

If we ignore multiple scattering and consider only single scattering for error calculation, Eq. 1 can be written in lowest order as:

$$
\frac{P(z)}{P_{0}}=1-s_{t}[1-G(z)]
$$


From Eq. 3 we can relate the standard deviation of the measured correlation function $\sigma_{G}$, to the standard deviation of the measured $\frac{P}{P_{0}}$ by:

$$
\sigma_{G}=\frac{\sigma_{P}}{P_{0}}
$$
unit time. If the transmission of the polarization analyzer for the up and down-spin states with no sample, are $T_{0}^{u}$ and $T_{0}^{d}$, respectively, then the measured beam polarization, $P_{0}^{\prime}$, is given by

$$
P_{0}^{\prime}=P_{0} \frac{T_{0}^{u}-T_{0}^{d}}{T_{0}^{u}+T_{0}^{d}}
$$

We can write a similar expression for the measured polarization, $P^{\prime}$, in the presence of a sample, where the measured polarization of the neutron beam is $P$, as

$$
P^{\prime}=P \frac{T^{u}-T^{d}}{T^{u}+T^{d}}
$$

Note that we have made an explicit distinction between the transmission without $\left(T_{0}^{u}\right.$ and $\left.T_{0}^{d}\right)$

177 states (i.e. that the analyzer is one that is designed to transmit spin-down neutrons), we can 


$$
\frac{P^{\prime}}{P_{0}^{\prime}}=\frac{P}{P_{0}}\left(\frac{1+\frac{T^{u}}{T^{d}}-\frac{T_{0}^{u}}{T_{0}^{d}}}{1-\frac{T^{u}}{T^{d}}+\frac{T_{0}^{u}}{T_{0}^{d}}}\right) .
$$

Evidently, if the ratio of the up to down transmission is the same for both the blank and the sample, the measured ratio of polarizations will be equal to $\frac{P}{P_{0}}$. However, if they are not, the term in parentheses in Eq.7 is not equal to unity and an error results.

As demonstrated by the computer simulations that follow, the transmission for up-spin state neutrons can vary by as much as $30 \%$ with both neutron wavelength and divergence angle and likewise up to $50 \%$ for down-spin state neutrons. Clearly this can lead to considerable error in determining $\frac{P}{P_{0}}$. In reality, however, neutrons are not all incident at the same divergence angle on the analyzer even without interacting with a sample so the effect in a real experiment is seldom as dramatic as our simulations might suggest. On the other hand, using the polarization analyzer that we simulate in this paper, we have observed errors in $\frac{P}{P_{0}}$ that translate into errors of more than 0.1 in the value of $G(z)$ at some values of the spin echo length, z. Given that $G(z)$ typically has a peak-to-trough amplitude of slightly greater than unity, this is a large error. It was errors of this sort that prompted the calculations presented here.

\section{III.Computer Simulation of the Multi-Channel Polarizer}

The computer simulations were carried out using the neutron ray-trace simulation package $\operatorname{McStas}^{24,25}$ version 2.0 which uses Monte Carlo methods to integrate over the relevant parameters for the instrument being modeled. It is worth pointing out that Dewhurst ${ }^{26}$ in 2012 provided a general approach for understanding such supermirror devices. His approach 
provides a useful framework for understanding results of detailed Monte Carlo calculations

$201 \quad$ such as those we present below.

202 Figure 1 gives a schematic of the supermirror polarizer device considered in this work. The 203 McStas software parameterizes reflectivity profiles for both nickel mirrors and supermirrors 204 by the following expression ${ }^{25}$ :

205

$$
R(Q)=\left\{\begin{array}{lr}
R_{0} & \text { if } Q<Q_{c} \\
\frac{1}{2} R_{0}\left(1-\tanh \left[\frac{Q-m Q_{c}}{W}\right]\right)\left(1-\alpha\left(Q-Q_{c}\right)\right) & \text { if } Q>Q_{c}
\end{array}\right.
$$

where $R(Q)$ is the mirror reflectivity as a function of the neutron momentum transfer $Q$, defined by $Q=4 \pi \sin (\theta) / \lambda$ where $\theta$ is the angle between the incident neutron and the reflecting plane and $\lambda$ is the neutron wavelength. The reflectivity profile represents the probability that a neutron will be reflected and thus the range of the function is between 0 and 1. Values of $R_{0}, Q_{c}, m, \alpha, W$ are given in the Table 1 for the nickel mirrors used for the channel walls and for the Fe-Si supermirrors. As described in the previous section, the Fe-Si supermirror has a different set of values for up-spin and down-spin neutrons and is designed to transmit a highly polarized beam of neutrons in the down-spin state. Nickel mirrors also have slightly different values for neutrons with different spins. $Q_{c}$ is calculated using data tabulated in Reference 23 . The reflectivity profiles calculated by using the values from Table 1 in Eq. 8 are shown in Fig. 3.

\begin{tabular}{|c|c|c|c|c|}
\hline & $\begin{array}{c}\text { Ni Mirror } \\
(+)\end{array}$ & $\begin{array}{c}\text { Ni Mirror } \\
(-)\end{array}$ & Fe-Si Supermirror (+) & Fe-Si Supermirror (-) \\
\hline$R_{0}$ & 1 & 1 & 1 & 1 \\
\hline$Q_{\mathrm{c}}$ & 0.02 & 0.0234 & 0.0108 & 0.0108 \\
\hline
\end{tabular}




\begin{tabular}{|c|c|c|c|c|}
\hline$m$ & 1 & 1 & 4.26 & 1 \\
\hline$\alpha$ & 0 & 0 & 5 & 0 \\
\hline$W$ & 0 & 0 & 0 & 0 \\
\hline
\end{tabular}

Table 1. Parameters for Ni mirror and Fe-Si supermirror reflectivity profiles. (+) refers to upspin state neutrons and (-) to down-spin state neutrons.

In order to isolate the performance of the multi-channel polarizer we did not simulate an entire SESANS instrument but rather we chose to model a simpler configuration consisting of a neutron source, an aperture, two channels of the multi-channel polarizer, and several virtual neutron detectors capable of capturing the flux, beam profile, and polarization of the neutron beam passing through the device. Figure 2 shows a schematic diagram of the model simulated. The neutron source is a $6.26 \mathrm{~mm} \times 6.26 \mathrm{~mm}$ square and neutrons are generated uniformly over its surface with a flat distribution of wavelength between $0.75 \AA$ and $21.5 \AA$. The source, double-channel supermirror device, and detectors are centered on the mid-line of the double-channel supermirror polarizer as shown in Fig. 2. A pinhole, which has the same dimensions as the source is located directly in front of the two channels of the supermirror polarizer so that the entrance is fully illuminated but all neutrons that would not enter the 33 device are blocked. The distance between the source and the entrance to the supermirrors polarizer is set initially to $2 \mathrm{~m}$. Neutrons are uniformly distributed from the source to the pinhole. Detectors (monitors in McStas terminology) are placed $5 \mathrm{~mm}$ before and after the device to record the neutron beam characteristics. For the majority of the simulations, the neutrons generated by the source were either in the up-spin state $(+)$ or the down-spin state (- 

unless otherwise indicated.

The transmission of the up and down-spin states as a function of neutron wavelength is shown in Fig. 4. For all plots we used a bin size of $0.5 \AA$, with the first point at $0.75 \AA$. For sufficiently short wavelength neutrons, the Fe-Si supermirrors transmit nearly all neutrons of both spin states because the incident angle exceeds the critical value. In addition, only those neutrons incident at very small angles on the Ni mirror surfaces are reflected. As a result, the transmission reaches a value of around $80 \%$ for both spin states for a wavelength around 0.75 $\AA$ (the first point). As the wavelength increases, transmission of the down-spin state (red) approaches unity with the exception of a dip at around $6 \AA$ while the transmission of the upspin state (blue) is generally low, as required for a good polarizer. Interestingly, nearly periodic oscillations are observed in the up-spin state transmission.

These oscillations arise from the drop in the up-spin reflectivity of the Fe-Si supermirror as Q increases from $0.0108 \AA^{-1}\left(Q_{c}\right)$ to $0.046 \AA^{-1}$ (critical edge) corresponding to a non-zero value of $\alpha$ (see reflectivity profile in Fig. 3 and their definitions in Eq. (8) and Table 1). The higher the value of $\alpha$, the lower the up-spin reflectivity at the supermirror critical edge. To investigate this further, we performed additional simulations for various values of the supermirror parameter $\alpha$. From Eq. 8 we see that as $\alpha$ decreases, the value of the reflectivity at the up-spin state critical edge increases, reaching a maximum value of unity when $\alpha=0$. 
Figure 5 shows the up-spin state transmission for the values of $\alpha$ simulated. Clearly, the oscillations always exist as long as $\alpha \neq 0$ and their amplitude grows with increasing $\alpha$.

The dip observed in the transmission of the down-spin state neutrons at around $6 \AA$ can be understood by studying the diagram presented in Fig. 6. For simplicity only the upper half of the device is considered. With the source size and distance used in the simulations, all neutrons have an incident angle, $\theta$, relative to the Fe-Si supermirror that lies between 0.0031 and 0.0071 radians. These values are represented by the horizontal lines (green and blue) in and the nickel walls before exiting. The value of $Q_{c}$ for down-spin neutrons incident upon the Fe-Si supermirror is $0.0108 \AA^{-1}$. Thus the critical angle, $\theta_{c}$, as a function of the neutron wavelength is given by

$$
\theta_{c}=\frac{0.0108 * \lambda}{4 \pi} \text { radians }
$$
and indicated by the red line in Fig. 6. Any neutrons whose incident angle and wavelength 272 lie above the red line will be transmitted through the supermirror; neutrons below the red line 273 will be reflected by the supermirror towards the upper Ni mirror in Fig. 1. The angle of 274 incidence of these neutrons on the upper Ni mirror will be $\theta_{N i}=\theta+\theta_{S M}$ where $\theta_{\mathrm{SM}}$ is the 275 angle between the Fe-Si supermirror and the Ni mirror. If this angle is less than the critical 276 angle for nickel, the neutron will be reflected from the nickel wall. The cyan line in Fig. 6 is represents the maximum angle for reflection from the nickel wall of the channel and is drawn 278 according to the equation

$$
\theta^{\prime}=\frac{0.02 * \lambda}{4 \pi}-\theta_{\mathrm{SM}}
$$


Any neutron whose incident angle on the supermirror $(\theta)$ is less than $\theta^{\prime}$ given by Eq. (10) will be reflected from the Ni mirror. Furthermore, $\theta_{S M}$ is determined from the dimensions of the device by the following expression them in the triangular area in Fig. 6 bounded by the red, green, blue and cyan lines will be reflected from the Fe-Si supermirror but will not be reflected from the Ni mirror and will therefore be absorbed giving rise to the dip observed at around $6 \AA$.

288

To confirm that it is possible to avoid this feature in down-spin state transmission, we performed two additional series of simulations. First, the length of the device was varied while holding with the channel width constant at $3.13 \mathrm{~mm}$. As a result, the observed dip in the down-spin state transmission moved to shorter wavelength with increasing length and eventually disappeared for device lengths greater than $1.5 \mathrm{~m}$, as shown in Fig. 7. Figure 8, which is analogous to Fig. 6, represents the various device lengths simulated. These plots show why the length of the device matters: the bounded region which includes the down-spin state neutrons that are absorbed moves to shorter wavelength as the device is made longer and thus the dip in the transmission becomes irrelevant because it moves out of the useful wavelength range of the analyzer. We also investigated the effect of tilting the device (about the $+y$ direction where $+z$ points in the direction the neutrons are traveling) such that the average incident neutron trajectory is no longer parallel to the Ni mirror walls of each channel. These simulations were performed, 
using only one channel of the device shown in Fig. 1 by tilting the device in the $+y$ and $-y$ direction. The resulting transmission of the down-spin state is shown in Fig. 9a and Fig 9b. The device length is set to $0.57 \mathrm{~m}$ for this simulation. We see that as the positive tilt angle increases the dip moves to longer wavelength and eventually disappears. Once again, this behavior can be understood from Fig. 6. Tilting the device corresponds to moving the two horizontal lines upwards on the figure while keeping their separation constant. Hence, the bounded area which defines the region from which neutrons are absorbed not only moves to longer wavelengths but also becomes smaller. A similar conclusion can be drawn for negative tilt angles.

312 It is the angle dependent transmission of the device that has the most serious consequences

313 for SESANS experiments. As pointed out earlier, the spatial and angular distribution of 314 neutrons scattered by the sample is different from that of the unscatterred beam. Thus, if the 315 polarization analyzer has a transmission efficiency that varies with position and angle, $P$ and $316 \quad P_{0}$ may be measured with different effective transmission functions and this, as we showed in 317 Eq. (7), may create an error. The error will be completely dependent on the details of the sample scattering. For this reason, no set of general corrections can be derived and the only solution is to use a polarization analyzer with a uniform transmission.

321 We found that the performance of the multichannel device is very sensitive to a number of 322 the mirror parameters in Table 1, especially the critical angle, $Q_{c}$, and the width of the 323 "cutoff" of the reflectivity at the critical edge, $W$. To investigate this sensitivity, we 324 performed several more simulations. In the preceding simulations we used a unrealistic 325 value of $W=0$, giving an extremely sharp cutoff of the reflectivity profile. This is never the 
case for real supermirrors and the value of $W$ is very sensitive to the details of how supermirrors are fabricated. Figure 10 shows the transmission of down-spin neutrons as the value of $\mathrm{W}$ is increased for both the Fe-Si supermirrors and the Ni mirrors. A series of curves with nonzero values for $W$ are shown, with $W=0.003$ being typical for Fe-Si supermirrors. As $W$ becomes larger, additional dips in the transmission develop at $\lambda=13 \AA$ value for $W$ gives rise to a tail extending the reflectivity profile slightly beyond the critical edge. When down-spin state neutrons intersect the supermirror with momentum transfers in the region where the reflectivity is decreasing rapidly, some of the neutrons will be reflected and eventually absorbed by the Ni mirror creating the dip we see in Fig. 10 at $13 \AA$ and $19 \AA$.

The value for the critical angle, $Q_{c}$, used for the down-spin state neutrons incident on the Fe-Si supermirror is the same as that for Si. However, there is slight difference between the down-spin state scattering length of $\mathrm{Fe}$ and $\mathrm{Si}$, both of which are components of the supermirror coating. Without detailed simulation or measurement of the supermirror reflectivity all that we really know is that down-spin $Q_{c}$ must be between that for Fe and Si. Accordingly, in Fig. 11 we plot a series of spin-

\section{Conclusion and Discussion}

346 We have shown that spatial and angular variations of the transmission function of a neutron 347 polarization analyzer used for SESANS can induce significant systematic errors into the 348 values of the correlation function deduced from the measurement. We have performed a series of simulations on the multi-channel polarizer used in our recent SESANS 
measurements. Our results show that the performance of the multi-channel polarizer is sensitive to many of the polarizer's parameters including length, width, tilt angle and mirror parameters, etc. While our results apply in detail only to a particular device, they do raise a concern about the functioning of any polarization analyzer used for SESANS that involves mirror reflections in a complex device whose properties inevitably change with wavelength. Such devices should be simulated before they are used for SESANS to avoid surprises in operation. Dalgliesh ${ }^{27}$ carried out such detailed simulations for the polarizing bender used on

\section{Acknowledgement}

This work was sponsored by the Scientific User Facilities Division, Office of Basic Energy Sciences, United States Department of Energy. Oak Ridge National Laboratory is managed by UT-Battelle, LLC, for the US Department of Energy (DOE) under contract No. DEAC05- 00OR22725. Roger Pynn acknowledges support from grant No. DE-FG0209ER46279 from the U.S. Department of Energy through its Office of Basic Energy Sciences, Division of Material Science and Engineering. 
375 1. C. G. Shull, E. O. Wollan and W. C. Koehler, Phys Rev 84 (5), 912-921 (1951).

$376 \quad 2 . \quad$ F. Mezei, Z Phys 255 (2), 146-\& (1972).

377 3. P. Courtois, B. Hamelin and K. H. Andersen, Nucl Instrum Meth A 529 (1-3), 157-161 (2004).

378 4. T. G. Walker and W. Happer, Rev Mod Phys 69 (2), 629-642 (1997).

$3795 . \quad$ K. H. Andersen, R. Chung, V. Guillard, H. Humblot, D. Jullien, E. Lelievre-Berna, A. Petoukhov and

F. Tasset, Physica B 356 (1-4), 103-108 (2005).

381 6. F. Mezei, Commun Phys 1 (3), 81-85 (1976).

382 7. F. Mezei and P. A. Dagleish, Commun Phys 2 (2), 41-43 (1977).

383 8. W. C. Chen, T. R. Gentile, C. B. Fu, S. Watson, G. L. Jones, J. W. Mclver and D. R. Rich, J Phys Conf Ser 294 (2011).

9. X. Tong, C. Y. Jiang, V. Lauter, H. Ambaye, D. Brown, L. Crow, T. R. Gentile, R. Goyette, W. T. Lee, A. Parizzi and J. L. Robertson, Rev Sci Instrum 83 (7) (2012).

10. A. loffe, E. Babcock, S. Mattauch, V. Pipich, A. Radulescu and M. S. Appavou, Chinese J Phys 50 (2), 137-154 (2012).

11. E. Lelievre-Berna and A. loffe, Neutron News 20 (1), 33 (2009).

12. V. Lauter, H. Ambaye, R. Goyette, W. T. H. Lee and A. Parizzi, Physica B 404 (17), 2543-2546 (2009).

13. T. Keller, T. Krist, A. Danzig, U. Keiderling, F. Mezei and A. Wiedenmann, Nucl Instrum Meth A 451 (2), 474-479 (2000).

14. N. Niimura, Basic Life Sci 64, 107-113 (1996).

15. W. G. Bouwman, R. Pynn and M. T. Rekveldt, Physica B 350 (1-3), E787-E790 (2004).

16. X. Li, B. Wu, Y. Liu, R. Pynn, C. Y. Shew, G. S. Smith, K. W. Herwig, J. L. Robertson, W. R. Chen and L. Liu, J Phys-Condens Mat 24 (6) (2012).

17. J. B. Hayter and H. A. Mook, Journal of Applied Crystallography 22, 35-41 (1989).

18. U. Rucker, E. Kentzinger, B. Toperverg, F. Ott and T. Bruckel, Appl Phys a-Mater 74, S607-S609 (2002).

19. M. Hino, H. Hayashida, M. Kitaguchi, Y. Kawabata, M. Takeda, R. Maruyama, T. Ebisawa, N. Torikai, T. Kume and S. Tasaki, Physica B 385-86, 1187-1189 (2006).

20. A. Stunault, K. H. Andersen, S. Roux, T. Bigault, K. Ben-Saidane and H. M. Ronnow, Physica B 38586, 1152-1154 (2006). Prince and J. Schadler, Nucl Instrum Meth A 671, 137-143 (2012).

22. V. G. Syromyatnikov, A. F. Schebetov, D. Lott, A. P. Bulkin, N. K. Pleshanov and V. M. Pusenkov, Nucl Instrum Meth A 634, S126-S129 (2011).

23. M. T. Rekveldt, J. Plomp, W. G. Bouwman, W. H. Kraan, S. Grigoriev and M. Blaauw, Rev Sci Instrum 76 (3) (2005).

24. K. Lefmann and K. Nielsen, Neutron News 10 (20) (1999).

25. P. Willendrup, E. Farhi and K. Lefmann, Physica B 350 (1-3), E735-E737 (2004).

26. C. D. Dewhurst, Nucl Instrum Meth A 683, 16-23 (2012).

27. R. Dalgliesh, Private Communication.

28. S. R. Parnell, K. Li and R. Pynn, Spin Echo Small Angle Neutron Scattering (SESANS) using a continuously pumped $3 \mathrm{He}$ analyzer (In Preparation). 


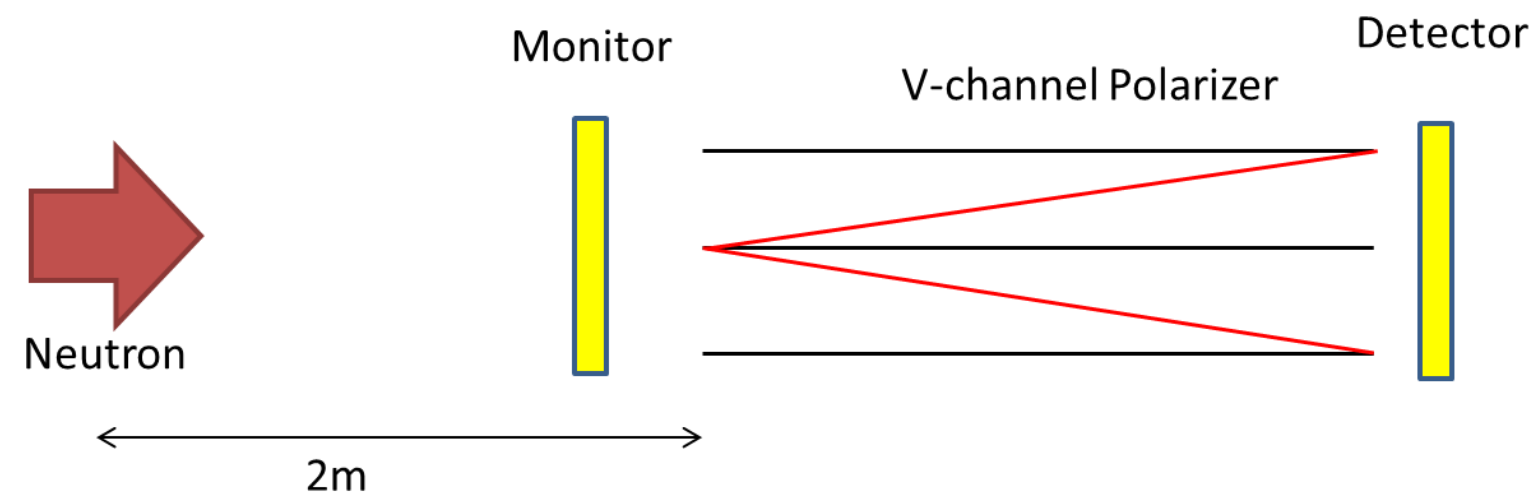

Figure 1: Schematic diagram of the simulated supermirror channel and the model simulated in McStas. Of the supermirror, horizontal lines represent Nickel coated glass, with absorbing material that prevents neutron transmission. The two tilted red lines represent polarizing supermirror coating on thin silicon, with different reflectivities for spin-up and spin-down neutrons. The width of $3.13 \mathrm{~mm}$, height of $82 \mathrm{~mm}$ and length of $570 \mathrm{~mm}$ are the default values. Some simulations are done by changing these parameters. In McStas simulations, the detector is capable of distinguishing between up-spin or down-spin neutrons. 


\section{Polarizer}

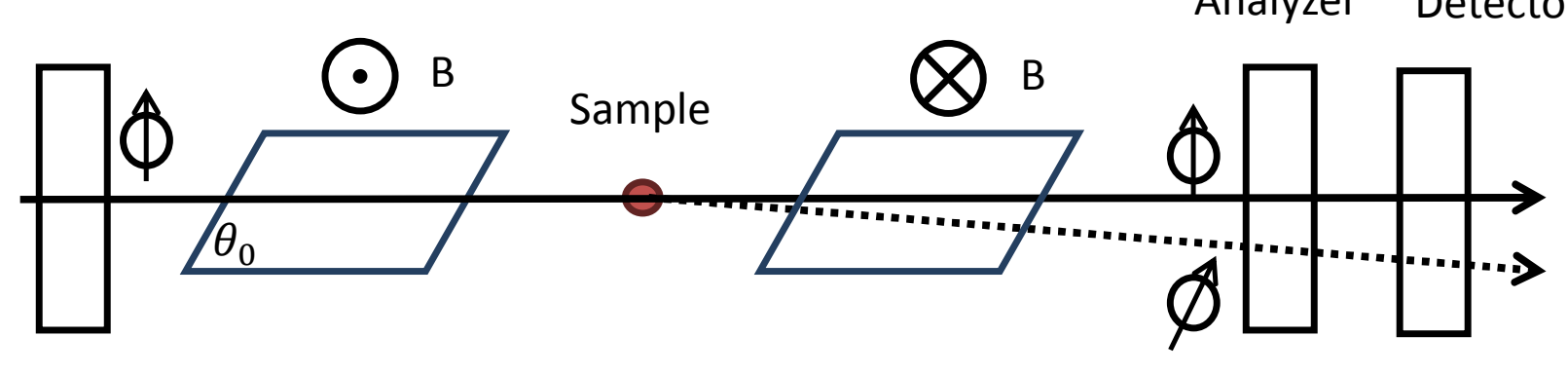

Figure 2: Diagram of a SESANS instrument. When the neutron trajectory is not deviated by the sample, the measured neutron polarization is the same as initial polarization; when neutrons are scattered by the sample, the final neutron polarization changes. Measurement of the polarization change directly gives the sample's real space correlation function. 


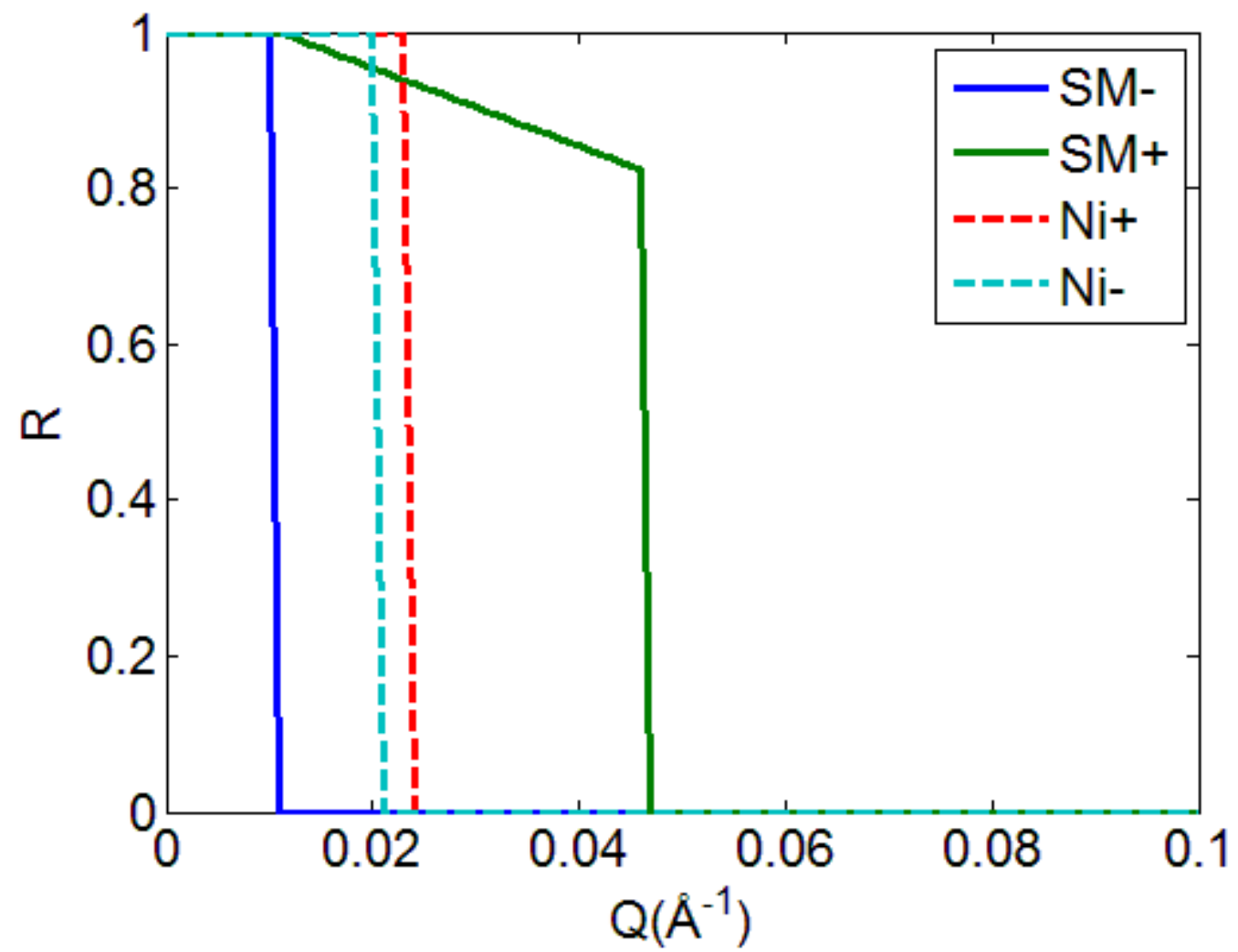

Figure 3. Reflectivity curves for the Nickel and Supermirror, calculated using Eq. 8 and the default parameters given in Table 1. 


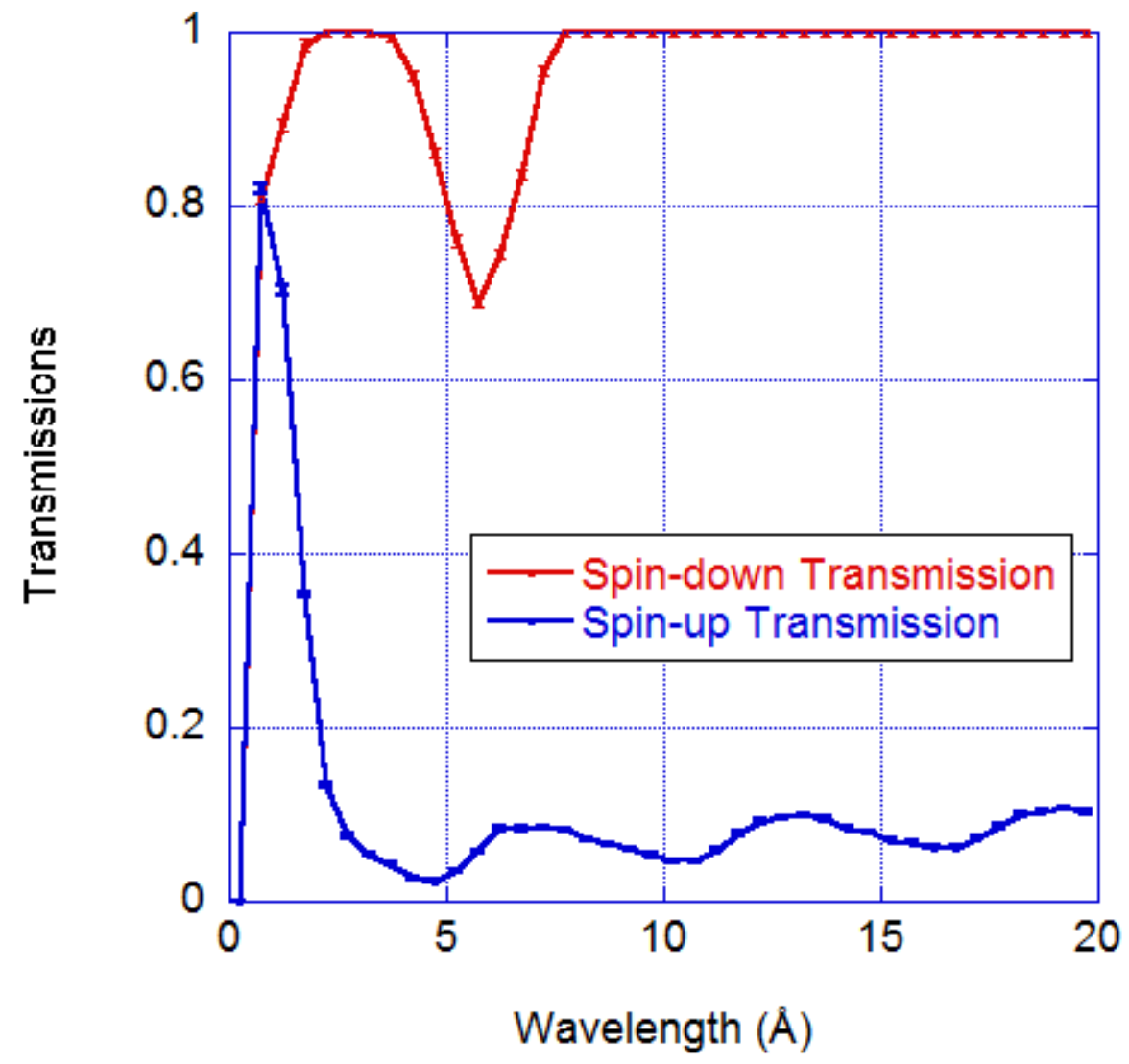

Figure 4. Simulated transmission through the multi-channel polarizer of incoming neutrons up-spin (blue) and down-spin (red). The bin size of the plot is $0.5 \AA$, with the first point at $0.75 \AA$. 


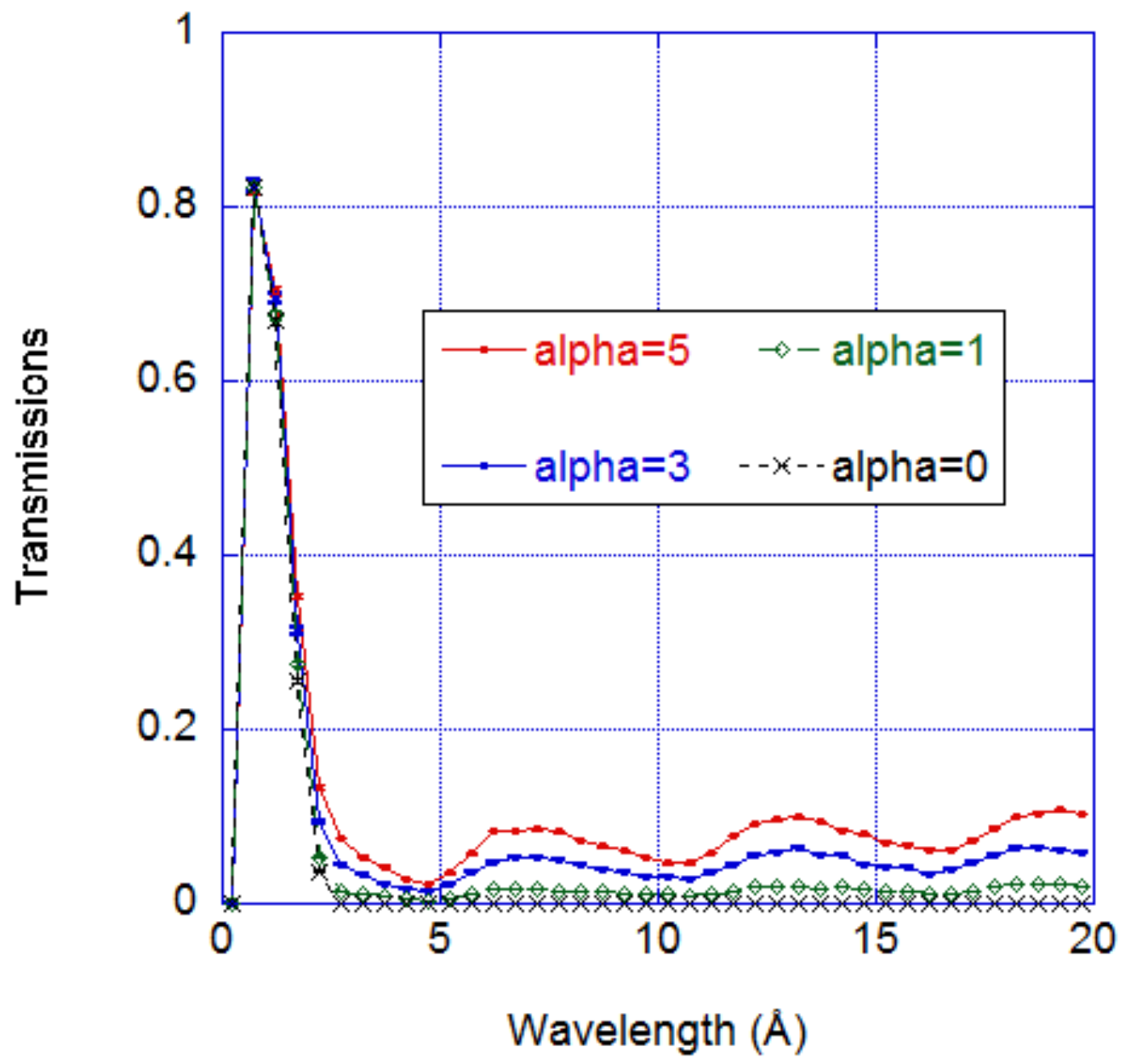

Figure 5: Simulated spin-up neutron transmission as a function of neutron wavelength for different values of the supermirror parameter $\alpha$. 


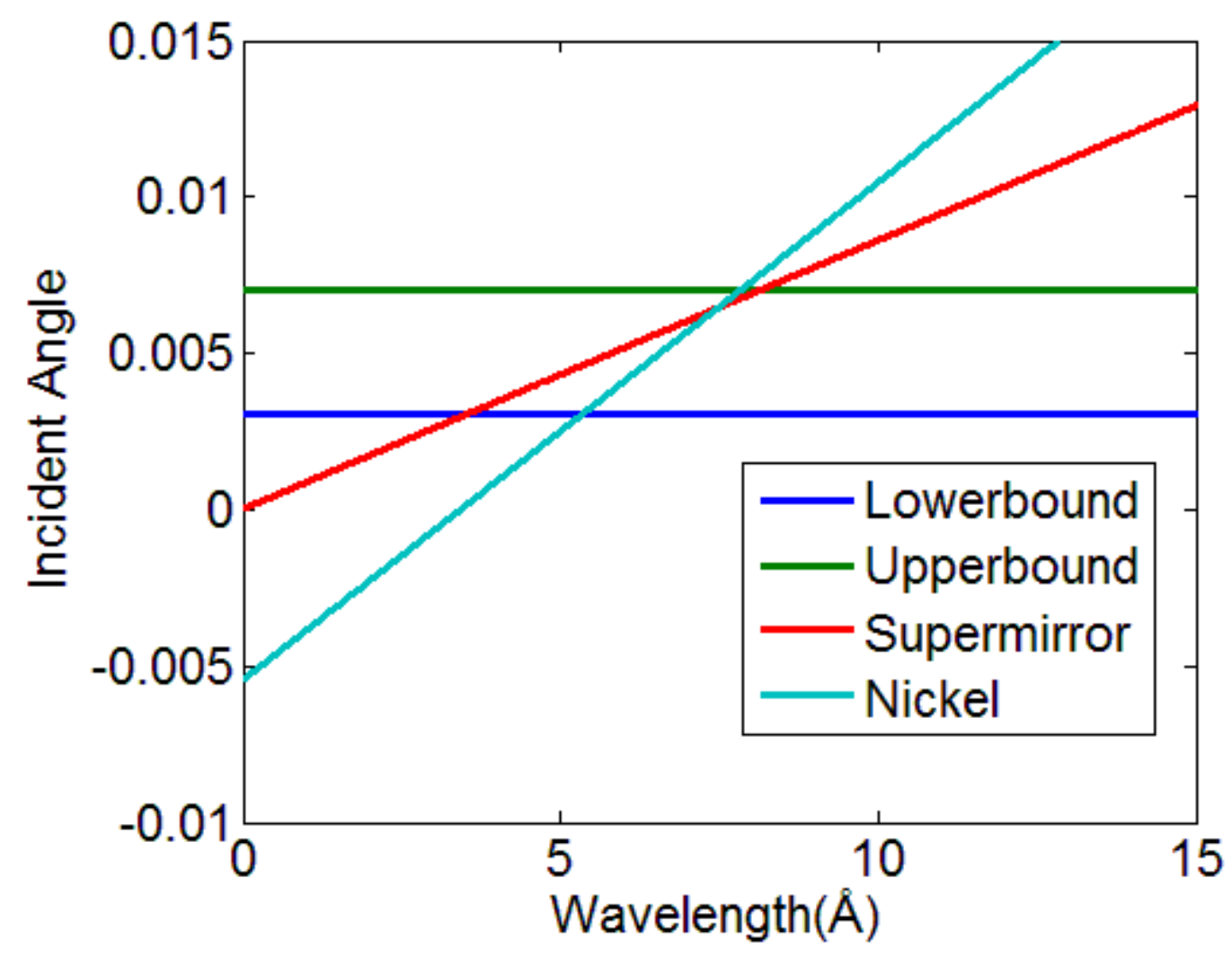

Figure 6. Plot of incident angle vs. wavelength for the upper channel shown in Figure 1. The blue and green horizontal lines are the maximum and minimum angles between a neutron trajectory and the nickel walls of the device. These values are defined by the size of the source and the width and length of the channel in the device. The red and cyan lines are explained in the text. The region bounded by all the lines represents spin-down neutrons that are reflected once from the supermirror but are NOT reflected by the nickel guide walls; these neutrons are lost from the down-spin transmitted beam, leading to a dip around $6 \AA$. 


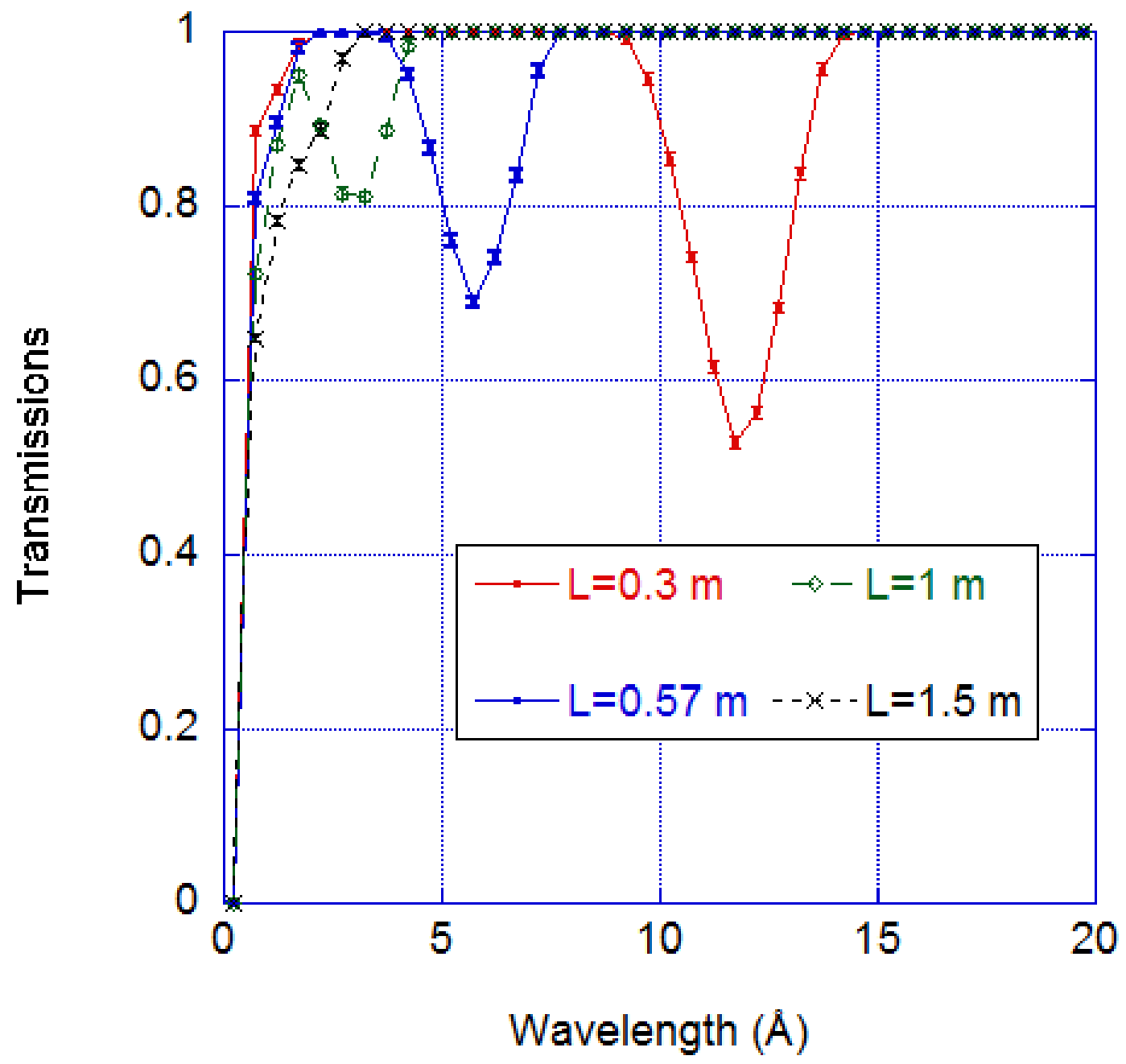

Figure 7. Simulated spin-down neutron transmission as a function of neutron wavelength for various device (channel) lengths. As the length increases, the dip in the transmission moves to shorter wavelength and eventually disappears for reasons described in the text. 

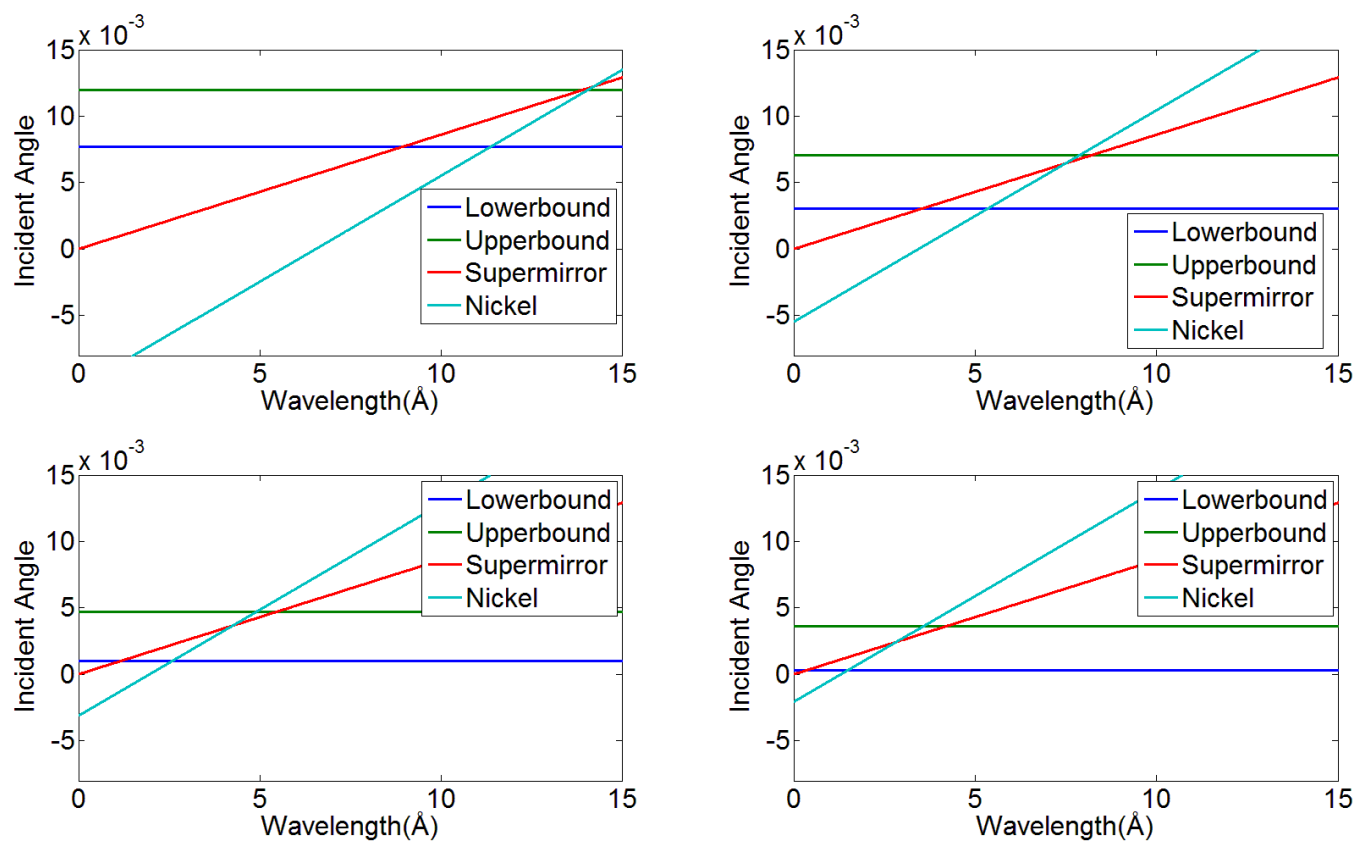

Figure 8. Incident angle vs. wavelength plots for various lengths of the device. The upper left panel is for $\mathrm{L}=0.3 \mathrm{~m}$; the upper right panel is for $\mathrm{L}=0.51 \mathrm{~m}$; the bottom left panel is for $\mathrm{L}=1 \mathrm{~m}$; the bottom right panel is for $L=1.5 \mathrm{~m}$. 

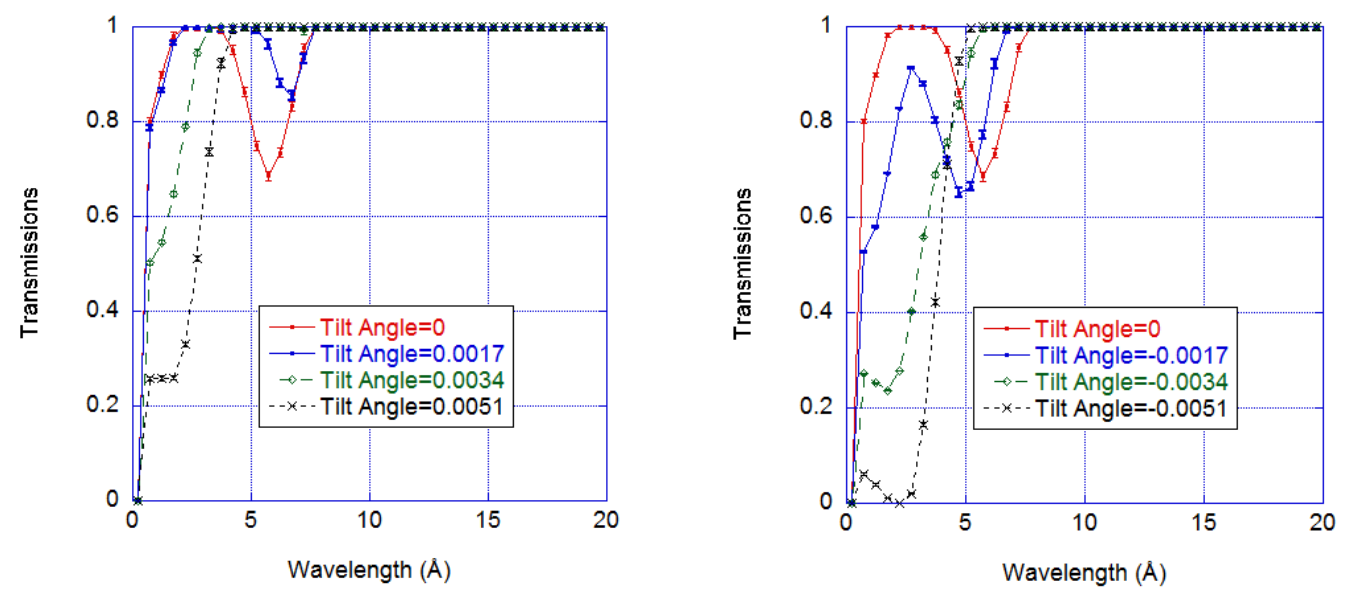

Figure 9. Simulated spin-down neutron transmission as a function of wavelength for various tilt angles (units in radians, 0.0017 radians $=0.1$ degree) of the device. As the positive tilt angle increases (left figure), the dip in the transmission moves to longer wavelength; as the negative tilt angle increases (right figure), the dip in the transmission moves to shorter wavelength. 


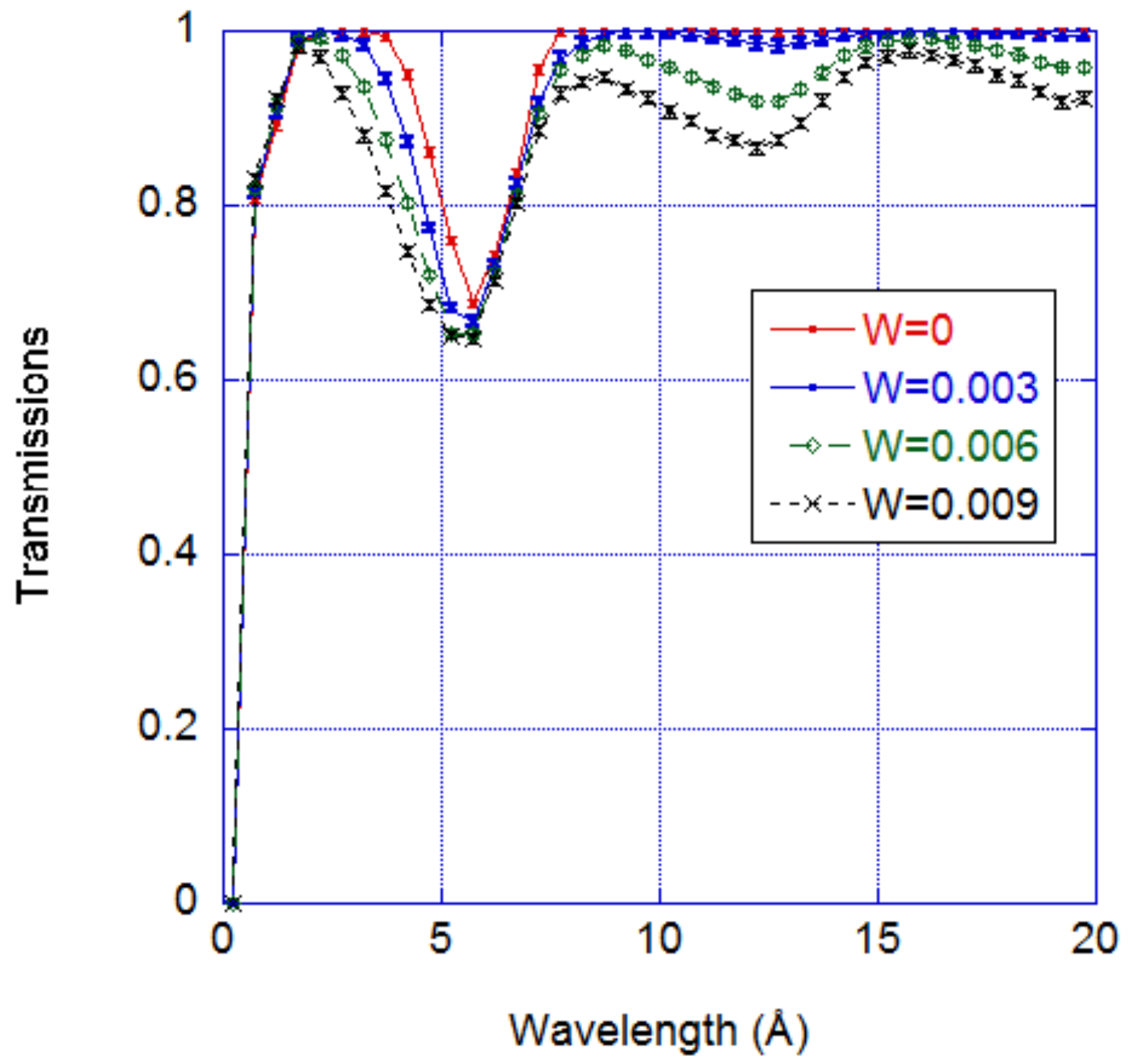

Figure 10. Simulated spin-down neutron transmission as a function of reflectivity parameter $W$. As $W$ increases, a new dip (centered at $13 \AA$ ) in the transmission appears and becomes larger, for reasons described in the text. 


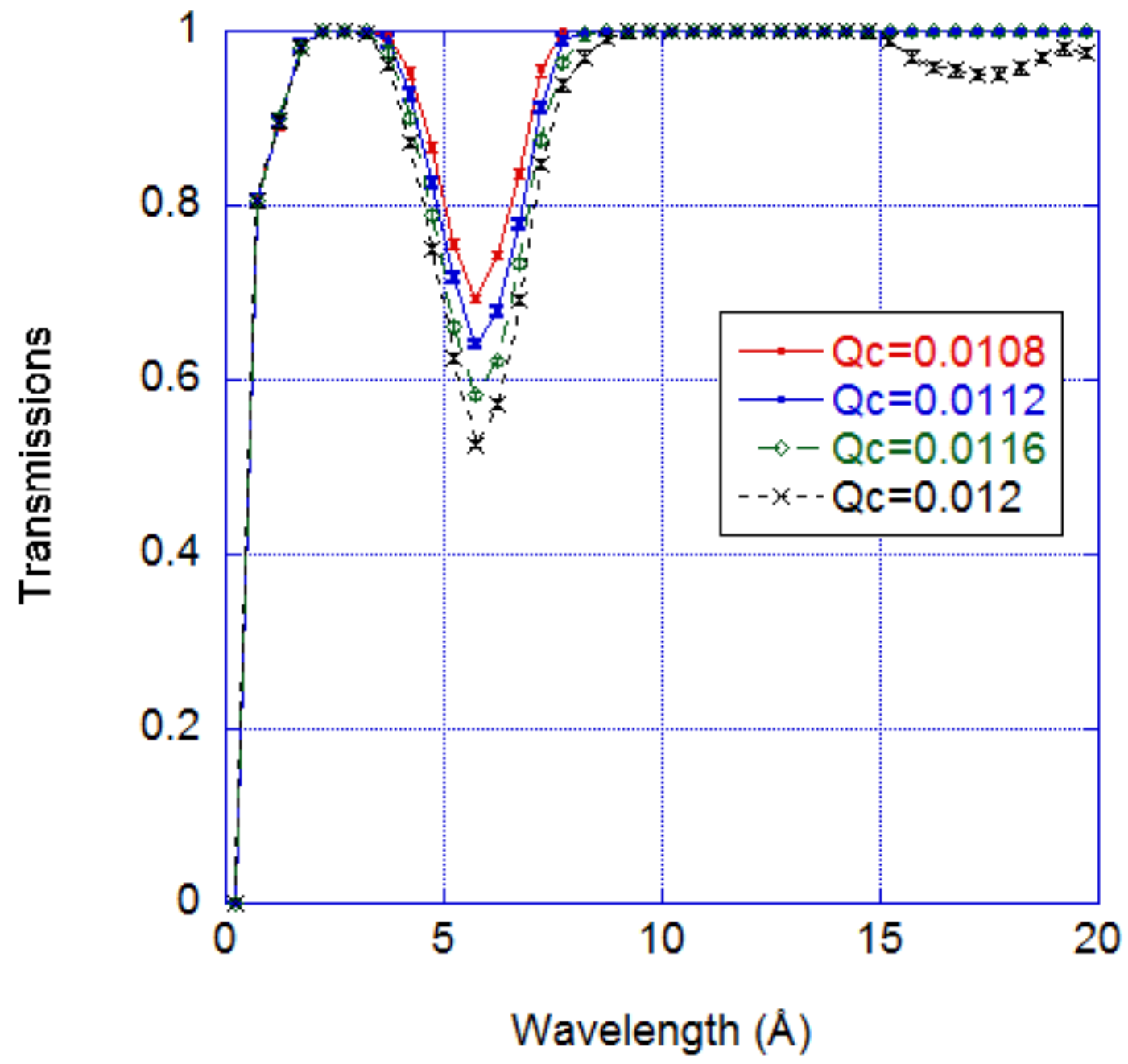

Figure 11. Simulated spin-down neutron transmission as a function of supermirror parameter $Q_{c}$ for spin-down neutrons. The transmission is very sensitive to the $Q_{c}$ value. As $Q_{c}$ increases, the dip at $6 \AA$ becomes larger. 